\title{
Evolution of Papillary Thyroid Carcinoma into Tall Cell Variant and TENIS Syndrome
}

\author{
Avik Chakraborty ${ }^{1}$, S.V. Kane ${ }^{2}$, Yogita Pawer ${ }^{1}$, and Sandip Basu ${ }^{1}$ \\ ${ }^{1}$ Radiation Medicine Centre, BARC, Mumbai, India; and ${ }^{2}$ Department of Pathology, Tata Memorial Hospital, Mumbai, India
}

\begin{abstract}
We herein report an unusual case of a 55-y-old woman with papillary carcinoma of the thyroid, who presented with multiple recurrences, with its subsequent evolution to tall cell variant and thyroglobulin-elevated negative iodine scintigraphy (TENIS) syndrome. During the course of the disease the lesions became non-iodine-concentrating with an increased proportion of tall cells and evidence of local and distant metastasis. Molecular analysis of the tissue specimen demonstrated $B R A F^{V 600 E}$ and $1582 \mathrm{M}$ mutations along with upregulation of tumor markers in metastatic tissue. The presence of $B R A F^{V 600 E}$ mutation and other markers warrants further investigation in future studies to define their precise implications for determining the aggressiveness and development into tall cell variant and TENIS.
\end{abstract}

Key Words: BRAF mutation; tall cell variant of PTC; lymph node metastasis; multiple recurrences

J Nucl Med Technol 2016; 44:255-258

DOI: $10.2967 /$ jnmt.116.181396

\section{A} mong papillary thyroid carcinoma (PTC) subtypes, the tall cell variant (TCV), characterized by tumor cell height 2 or 3 times the width, has frequently been associated with a more aggressive clinical course. There have been endeavors to clearly define the correlation between prognostic characteristics and molecular markers for TCV (1).

Mutations in the BRAF protooncogene have been predominantly detected in PTC (2). Though yet unclear, there may exist a correlation between the aggressiveness of TCV and the presence of the $B R A F^{V 600 E}$ gene mutation (3). The presence of the rare $B R A F$ mutation $\left(B R A F^{I 582 M}\right.$ ) in classic PTC histology (but not in TCV) has been reported. The influence of RET-PTC, PAX8-PPAR $\gamma$, mucin1 (MUC1), cyclin D1, galectin 3, mesothelioma1 (HBME1), and cytokeratin 19 (CK19), which play critical roles in the oncogenic transformation of classic and follicular variant of PTC, is reported to be equivocal in the TCV patients. Herein, we have investi-

\footnotetext{
Received Jul. 10, 2016; revision accepted Oct. 17, 2016.

For correspondence or reprints contact: Sandip Basu, Radiation Medicine Centre, BARC, C/O Tata Memorial Hospital Annexe, Parel, Mumbai-400012, India.

E-mail: drsanb@yahoo.com

Published online Nov. 10, 2016.

COPYRIGHT (c) 2016 by the Society of Nuclear Medicine and Molecular Imaging, Inc.
}

gated the relationship between the prognostic and diagnostic markers in TCV of PTC (with its evolution to thyroglobulinelevated negative iodine scintigraphy [TENIS] syndrome).

\section{CASE REPORT}

A 46-y-old woman with the diagnosis of PTC had undergone near-total thyroidectomy 10 y previously. Within 3 mo of the first surgery, a second operation was performed to remove the growth over the tracheal surface. Histopathology was suggestive of a metastatic lymph node from classic PTC. Five years after this surgery, the patient developed a large, palpable mass in the thyroid bed. Excision biopsy of the mass demonstrated metastatic deposits of PTC in lymph nodes. Because of the subsequent nodal recurrence, she was considered for empiric radioiodine therapy $(5,735$ $\mathrm{MBq})$. There was minimal response, and she underwent neck dissection for the recurrent mass (Fig. 1). Histopathology denoted the presence of PTC with left-side neck node metastasis. A distinct tall cell pattern was observed for the first time since initial diagnosis. Two years after this, another recurrence was detected, necessitating the surgical removal of the left cervical lymph node, which was found on histologic examination to be metastatic PTC of the tall cell pattern. Subsequently, she presented with multiple neck nodal recurrences over $6 \mathrm{y}$, which necessitated surgical removal. There was no radioiodine concentration in the tumor recurrences (commensurate with diagnosis of TENIS syndrome) during these surgical removals, and hence such radioiodine therapy was not considered. Wholebody ${ }^{18}$ F-FDG PET had shown avid uptake in the neck recurrences, as could be expected (Fig. 2); on the last occasion the $\mathrm{SUV}_{\max }$ was 10.3. Interestingly, distant metastases were not documented in the previous ${ }^{18}$ F-FDG PET studies. The thyroglobulin values were elevated at each recurrence and ranged from 23 to $340 \mathrm{ng} / \mathrm{mL}$. After the last surgery, the patient was treated with external-beam radiotherapy to the neck in view of multiple recurrences but later developed ${ }^{18} \mathrm{~F}-\mathrm{FDG}$-avid pulmonary metastases, with the largest nodule documented as $1.7 \mathrm{~cm}\left(\mathrm{SUV}_{\max }, 38.1\right)$.

\section{Histopathologic Finding}

The histologic diagnosis of the completion-thyroidectomy specimen showed solid tumors with a papillary pattern and no tall cells. At places, the papillae were closely packed. In 
FIGURE 1. ${ }^{131}$ I scan. High count neck and chest view of ${ }^{131}$ I whole-body scan during last recurrence obtained $72 \mathrm{~h}$ after oral administration of $148 \mathrm{MBq}$ of ${ }^{131}$ I demonstrates no abnormal focus in neck and chest, implying palpable neck nodes are non-iodine-concentrating (a feature of dedifferentiation in thyroid carcinoma).

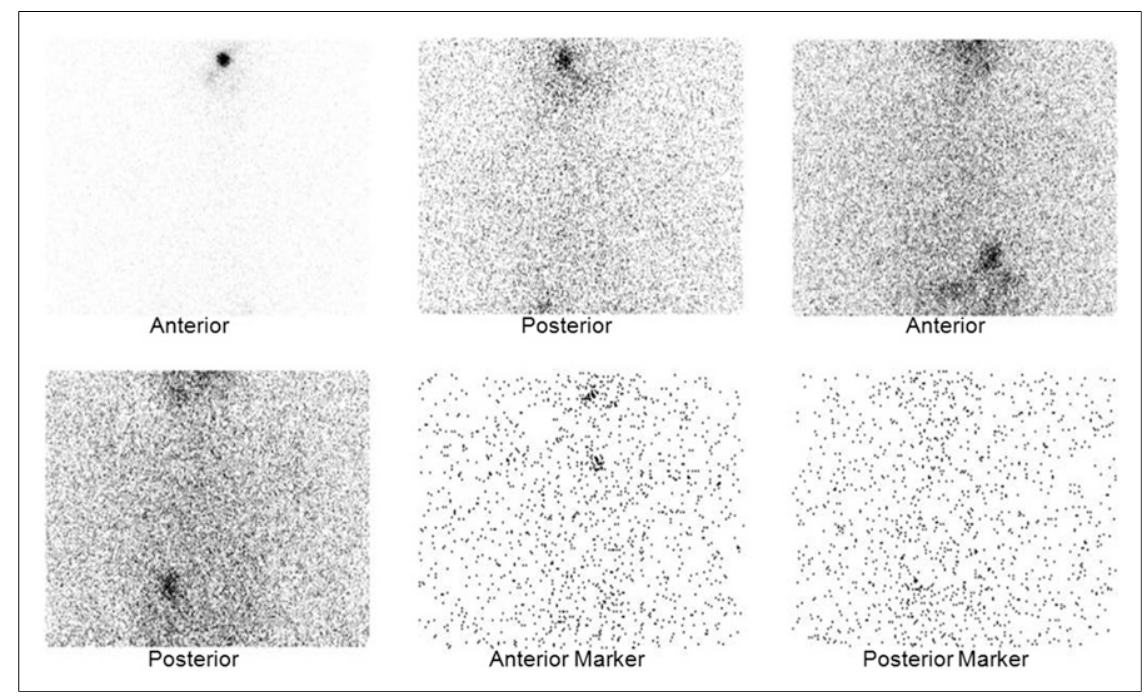

FIGURE 2. ${ }^{18}$ F-FDG PET scan. Wholebody ${ }^{18}$ F-FDG PET (coronal slices) images during time of last recurrence obtained $60 \mathrm{~min}$ after injection of 370 $\mathrm{MBq}$ of ${ }^{18} \mathrm{~F}-\mathrm{FDG}$ demonstrates multiple ${ }_{18}^{18} \mathrm{~F}-\mathrm{FDG}$-avid neck nodes $\left(\mathrm{SUV}_{\max }\right.$, 10.3). Last slide demonstrates ${ }^{18} \mathrm{~F}-\mathrm{FDG}-$ avid metastatic right pulmonary lesions $\left(\mathrm{SUV}_{\max }, 38.1\right)$.

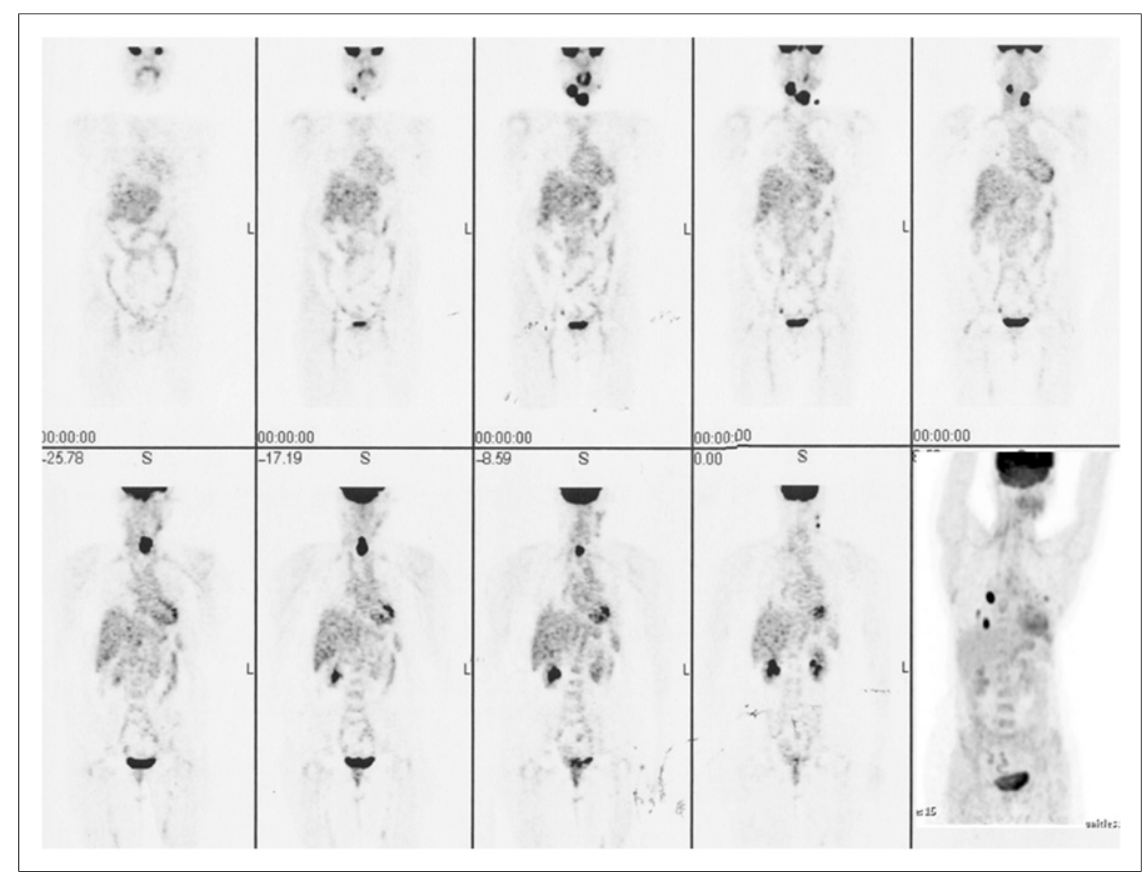

the metastatic recurrences, the papillae were covered by a single layer of tall columnar cells. The tall cell pattern was seen in $90 \%$ of the metastatic specimens but not in the primary tumor (Fig. 3A). The height of the tumor cell was 2-3 times the width. Aggressive features such as brisk mitotic activity, necrosis, and nuclear pleomorphism were seen (Fig. 3B).

In the surgical specimen from the 2010 recurrence, the nodes showed metastasis of PTC with tall cell morphology. Cystic changes and extranodal extension were also noted. The immunohistochemical markers cyclin D1, galectin 3, HBME1, and CK19 were unregulated in the recurrent metastatic specimen.

\section{Genetic Analysis}

Frozen thyroid tissue samples were used for extraction of DNA and RNA with QIAapm Kits (Qiagen) according to the manufacturer's instructions.

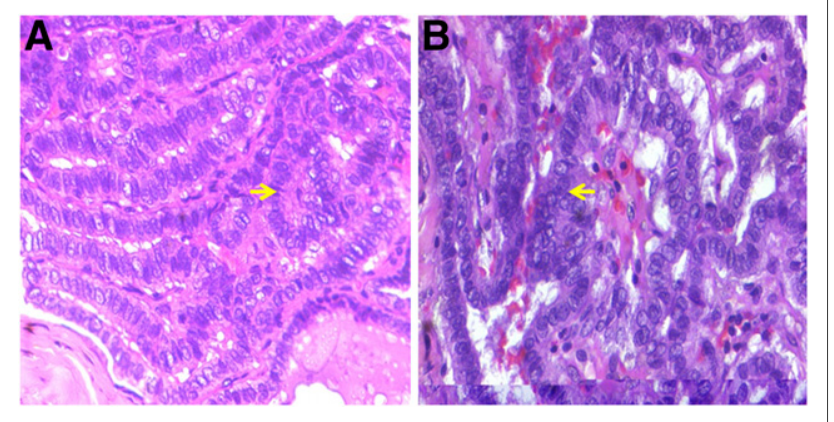

FIGURE 3. Hematoxylin and eosin sections (200x); TCV of PTC. (A) Note compactly arranged fingerlike projections with dense eosinophilic cytoplasm, overlapping nuclei, and pseudoinclusion (arrows). (B) Hematoxylin and eosin section (400x) shows papillary projections covered by tall cells (arrows). 


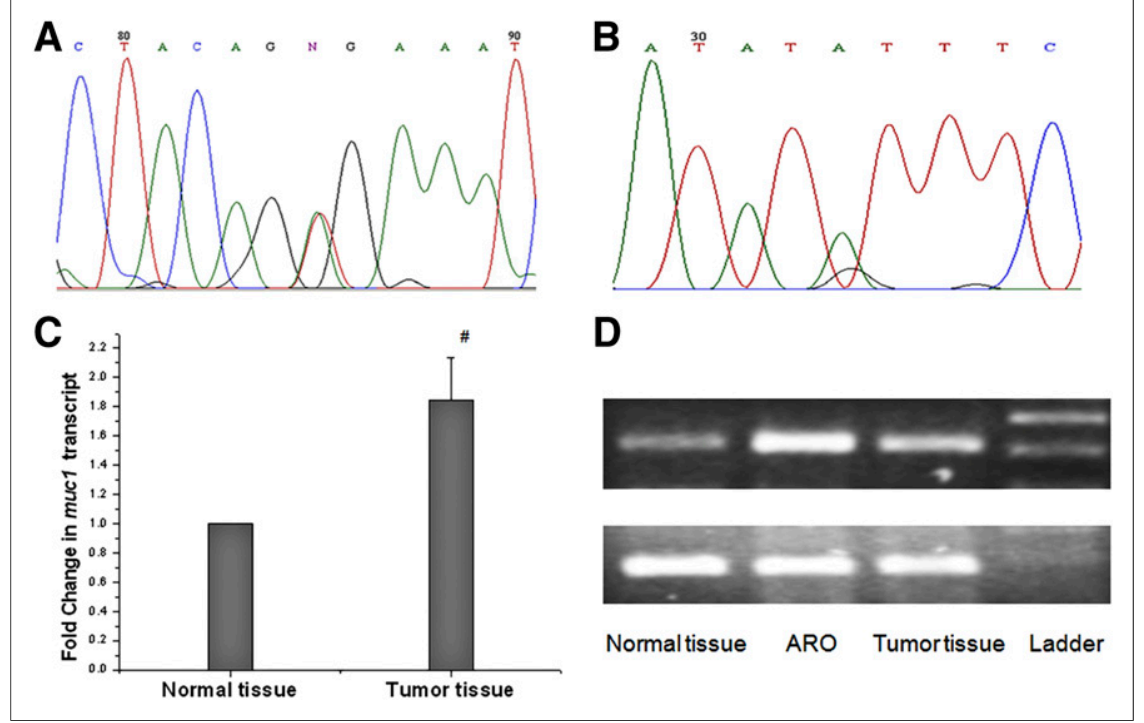

FIGURE 4. BRAF mutation. (A) Sequence chromatograph of tumor showing heterozygous BRAF T1799A mutation. (B) $B R A F^{1582 M}$ point mutation in same tissue specimen. (C) Real-time polymerase chain reaction: graph shows relative messenger RNA expression. Two-fold increase on MUC1 level is observed. (D) Semiquantitative real-time polymerase chain reaction: muc1 gene (upper) with respective $\beta$ actin genes (lower).
Exon 15 of the $B R A F$ gene was amplified as previously described using the following primers: $5^{\prime}$ TCATAATGCTTGCTCTGATAGGA3' and 5'GGCCAAAATTTAATCAGTGGA3' (4). The thyroid carcinoma cell lines ARO and WRO were used as positive and negative controls, respectively. Polymerase chain reaction products were sequenced in both directions using ABI Prism 377-18 and compared by the BLAST program (www.ncbi.nlm.nih.gov/BLAST), revealing $B R A F^{V 600 E}$ and $B R A F^{I 582 M}$ mutations (Figs. 4A and $4 \mathrm{~B})$.

Complementary DNA from tumor and adjacent normal tissue was analyzed with $\mathrm{MUC1}$ primers in SYBR green-based qPCR (Stratagene). The expression of MUC1 gene at the transcriptional level showed a 2-fold increase in comparison to the normal tissue (Figs. 4C and 4D).

\section{DISCUSSION}

In this patient, all metastatic tissue specimens collected at different time points showed the $B R A F^{V 600 E}$ and $B R A F^{I 582 M}$ mutations (Figs. 4A and 4B). According to the literature, the presence of a $B R A F$ mutation increases the risk of lymph node metastasis and the difficulty of treatment. This clearly indicates that the persistent presence of the constitutively expressing $B R A F$ gene may have a role in multiple recurrences (4).

Our study also revealed that multiple markers of tumor aggressiveness, including MUC1 (Figs. 4C and 4D), CK19, cyclin D1, galectin 3, and HBME1 (Fig. 5), were overexpressed in this case.

All these cell adhesion and cell cycle regulatory proteins are reportedly helpful for differential diagnosis of endocrine malignancy. A combined analysis of these markers for a diffuse expression pattern and intense cellular positivity may better predict a poor prognosis of TCV.

${ }^{18} \mathrm{~F}-\mathrm{FDG}$ PET is now considered an important modality for investigating patients with high thyroglobulin and no abnormal iodine concentration seen on whole-body radio- iodine scanning. ${ }^{18} \mathrm{~F}-\mathrm{FDG}$-avid disease is also associated with tumor aggressiveness and relative resistance to radioiodine therapy even in patients with differentiated thyroid carcinoma who demonstrate radioiodine concentration. It is logical to hypothesize that the aggressiveness of the disease in terms of clinical behavior could be related to the genetic mutation that is associated with aggressive features in differentiated thyroid carcinoma. The finding in this challenging case also supports this. We thus propose that there is a relationship between the BRAF genetic mutation in these lesions and their in vivo imaging and histologic features as observed in the present case study (5). We believe that the findings observed in our patient require

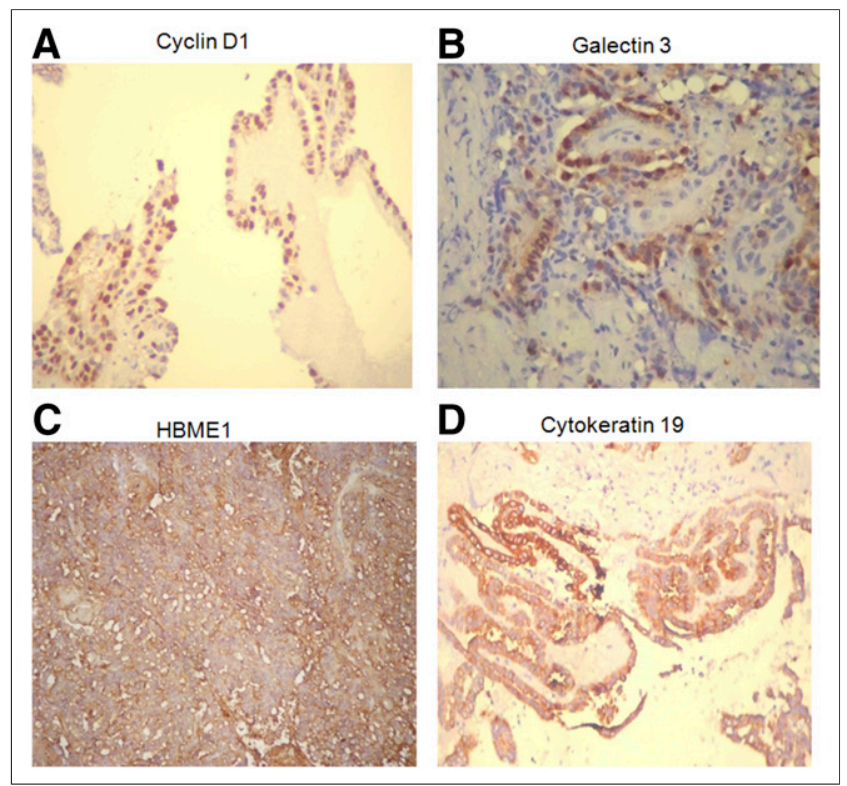

FIGURE 5. Immunohistochemical detection of different tumor markers (200x): Cyclin D1 (A), galectin-3 (B), HBME1 (C), and CK19 (D). 
further examination in future studies and that if a relationship is proven, further research with $B R A F$-specific therapies (e.g., BAY 43-9006, AMG 706, and vemurafenib) in this group of patients could be expanded.

\section{CONCLUSION}

The due progression and dedifferentiation of an aggressively behaving classic PTC were analyzed morphologically, genetically, histopathologically, and immunohistochemically. The histopathology, imaging features, and $B R A F^{V 600 E}$ mutation along with observation of rare $B R A F^{I 582 M}$ gene mutation in the setting of TCV warrant study of additional cases to understand the association of these markers with this rare histotype.

\section{DISCLOSURE}

No potential conflict of interest relevant to this article was reported.

\section{REFERENCES}

1. Michels JJ, Jacques M, Henry-Amar M, Bardet S. Prevalence and prognostic significance of tall cell variant of papillary thyroid carcinoma. Hum Pathol. 2007;38:212-219.

2. Trovisco V, Soares P, Sobrinho-Simões M. B-raf mutations in the etiopathogenesis, diagnosis, and prognosis of thyroid carcinomas. Hum Pathol. 2006;37:781-786.

3. Chakraborty A, Narkar A, Mukhopadhyaya R, Kane S, D’Cruz A, Rajan MG. Braf v600e mutation in papillary thyroid carcinoma: significant association with node metastases and extra thyroidal invasion. Endocr Pathol. 2012;23:83-93.

4. Trovisco V, Vieira de Castro I, Soares P, et al. Braf mutations are associated with some histological types of papillary thyroid carcinoma. J Pathol. 2004;202:247-251.

5. Basu S, Urhan M, Rosenbaum J, Alavi A. PET and PET/CT in the management of thyroid cancer. Methods Mol Biol. 2011;727:205-224. 